\title{
The first law simulation and second law analysis of a typical reciprocating air compressor
}

\author{
T. Armaghani ${ }^{1}$, S.A.R. Sahebi ${ }^{2}$ and H. Goodarzian ${ }^{1}$ \\ ${ }^{1}$ Department of Mechanical Engineering, Islamic Azad University, Mahdishahr Branch, Mahdishahr, Iran \\ ${ }^{2}$ Department of Mechanical Engineering, Islamic Azad University, Savadkooh Branch, Savadkooh, Iran
}

h.goodarzian@yahoo.com

\begin{abstract}
The importance of developing thermal systems that effectively uses energy resources such as natural gas is apparent. Effective use is determined with both the first and second laws of thermodynamics. In this paper, an exergy analysis was presented based on the first and the second laws of thermodynamics to study in-cylinder properties of the air in a typical reciprocating compressor at each crank angle. A computer program has been developed to study the full operation cycle of an air compressor. The simulation used to calculate the pressure and temperature field existing in realistic compressor chambers for various engine speeds. The results show a good agreement with previous studies where applicable.
\end{abstract}

Keywords: Reciprocating air compressor, Thermodynamics modeling, Exergy analysis.

Introduction

The exergy analysis is a tool to identify losses and destructions so that appropriate measures can be implemented to reduce the losses and destructions (Seyyedi et al., 2010). Energy cannot be destroyed. The idea that something can be destroyed is useful in analysis of Energy Systems. This idea does not apply to energy, however, but to exergy. Exergy of a thermodynamic system is the maximum theoretical useful work (shaft work or electrical work) obtainable as the system is brought into complete thermodynamic equilibrium with the thermodynamic environment while the system interacts with this environment only (Tsatsaronis, 2007; Gorji et al., 2010; Gorji \& Goodarzian, 2011).

Reciprocating air compressors are widely used in the industries in order to supply high pressure fluid streams. They are simple in principle and can accept wide variations in suction and discharge conditions. Their flexibility is combined with the minimum power per machine volume over the wide operating range (Hough, 1995; Sun \& Ren, 1995; Heywood, 1988; Weclas, 1998). Moreover, only reciprocating compressors can provide very high pressure ratios. Various modelling methods have been developed for reciprocating compressors thermodynamic analysis. These methods can be roughly classified in global models (Stouffs et al., 2001; Duprez et al., 2007; Navarro et al., 2007) and in differential models (Cezar et al., 2011) where the variables depend on time (or crank angle). In the global models, a limited number of algebraic equations are supposed to describe the reciprocating compressor thermodynamic behaviour. Several parameters have to be given a priori or to be identified experimentally. Among these, there is always at least one so called polytropic exponent. Very often, the physical phenomena that influence these parameters are not straightforward. Moreover this approach is open to criticism since the compression and the expansion processes are usually very different from polytrophic processes due to alternate gas-to-wall heat transfer. Furthermore, in these models, the influence of the residual fluid (that is the fluid which is contained in the clearance volume and which is not discharged) on the power consumption is generally not clearly identified. In the differential models, at least three nodes are considered, namely the suction plenum, the cylinder space and the discharge plenum. In some of these models (Ndiaye \& Bernier, 2010), the single node corresponding to the cylinder is replaced by a finite difference grid, so that the velocity field in the cylinder, and, ultimately, local gas-to-wall heat transfer can be better evaluated.

In this paper a simple global model has been developed to study the effect of engine speed on thermodynamics properties of a typical compressor. The model can predict the volumetric effectiveness, the specific work and the indicated efficiency of the reciprocating compressor for different operating pressure ratios. The model allows physical interpretation. In particular, the influence wall heat transfers are dealt with and the negative impact of the clearance volume is evaluated.

Thermodynamics modeling

As analytic functions cannot be used to describe engine processes, it is necessary to solve the governing equations on a step-wise basis.

The equation of state for an ideal gas is

$$
P V=m R T
$$

Taking the logarithm of both sides and differentiating with respect to crank angle gives

$$
\frac{1}{R}(P d V+V d P)=m d T+T d m
$$

It can be assumed that the internal energy is only a function of the temperature and the combustion process is modelled as a single zone. The first law of thermodynamics can be written in differential form for an open thermodynamic system. If changes in potential energy are neglected, then

$$
\begin{aligned}
& m_{C} C_{V}(d T)+C_{V} T_{C}(d m)=\partial Q-P d V+(d m) h_{i} \\
& +m_{i} C_{P}(d T)-(d m) h_{e}+m_{e} C_{P}(d T)
\end{aligned}
$$

Research article

CIndian Society for Education and Environment (iSee)
"Reciprocating air compressor" http://www.indjst.org
Armaghani et al. Indian J.Sci.Technol. 
With combining equation (2) and (3) and arranging it we get

$$
\begin{aligned}
& \left(1+\frac{C_{V}}{R}\right) P d V=\partial Q-\frac{C_{V}}{R} V d P+(d m) h_{i}+ \\
& m_{i} C_{P}(d T)-(d m) h_{e}+m_{e} C_{P}(d T)
\end{aligned}
$$

Introducing $C_{P}-C_{V}=R$ and $C_{P}=C_{V} K$ then rearranging equation (4) could be modified as below:

$\frac{d P}{d \theta}=\frac{K-1}{V} \frac{\partial Q}{\partial \theta}-\frac{K}{V} P \frac{d V}{d \theta}+\left(\frac{d m_{i}}{d \theta}\right) R K T_{i}$

$+m_{i} R K\left(\frac{d T_{i}}{d \theta}\right)-\left(\frac{d m_{e}}{d \theta}\right) R K T_{e}-m_{e} R K\left(\frac{d T_{e}}{d \theta}\right)$

This equation has been solved iteratively to predict in cylinder pressure in each crank angle. From the first law of thermodynamics for changing mass system we have

$\frac{d Q}{d \theta}+\frac{d m_{s}}{d \theta} h_{s}=\frac{d W}{d \theta}+\frac{d m_{d}}{d \theta} h_{c}+\frac{d\left(m_{c} u_{c}\right)}{d \theta}$

Where

$\frac{d W}{d \theta}=p_{c} \frac{d V_{c}}{d \theta} ; \quad h=u+p v$

Where $\mathrm{h}$ is enthalpy, $\mathrm{v}$ is specific volume and subscripts $\mathrm{c}, \mathrm{s}$ and $\mathrm{d}$ respectively refers to inside cylinder, suction and discharge and $v=\frac{V}{m}$ thus

$\frac{d\left(m_{c} u_{c}\right)}{d \theta}=\frac{d\left(m_{c} h_{c}\right)}{d \theta}-\frac{d\left(m_{c} p_{c} v_{c}\right)}{d \theta}=m_{c} \frac{d h_{c}}{d \theta}$

$+h_{c} \frac{d m_{c}}{d \theta}-p_{c} \frac{d V_{c}}{d \theta}-V_{c} \frac{d p_{c}}{d \theta}$

Substituting Eqn (7) into Eqn (6):

$\frac{d h_{c}}{d \theta}=\frac{1}{m_{c}}\left[\frac{d m_{s}}{d \theta}\left(h_{s}-h_{c}\right)+V_{c} \frac{d p_{c}}{d \theta}+\frac{d Q}{d \theta}\right]$

In addition,

$\frac{d h}{d \theta}=\left(\frac{\partial h}{\partial T}\right)_{v} \frac{d T}{d \theta}+\left(\frac{\partial h}{\partial v}\right)_{T} \frac{d v}{d \theta}$

$\frac{d p}{d \theta}=\left(\frac{\partial p}{\partial T}\right)_{v} \frac{d T}{d \theta}+\left(\frac{\partial p}{\partial v}\right)_{T} \frac{d v}{d \theta}$

Substituting Eqn (8) into Eqn (9) gives

$$
\begin{aligned}
& \frac{d T_{c}}{d \theta}=\frac{1}{\left[\left(\frac{\partial h_{c}}{\partial T_{c}}\right)_{v}-v_{c}\left(\frac{\partial p_{c}}{\partial T_{c}}\right)_{v}\right]} \times \\
& \left\{\frac{1}{m_{c}}\left[\frac{d m_{s}}{d \theta}\left(h_{s}-h_{c}\right)+\frac{d Q}{d \theta}\right]+\left[v_{c}\left(\frac{\partial p_{c}}{\partial v_{c}}\right)_{T}-\left(\frac{\partial h_{c}}{\partial v_{c}}\right)_{T}\right] \frac{d v_{c}}{d \theta}\right\}
\end{aligned}
$$

\section{Heat transfer equation}

For an air compressor, the heat transfer is given by Heywood (1988):
Vol. 5 No. 3 (Mar 2012)

ISSN: 0974- 6846

$Q=h A\left(T_{g}-T_{w}\right)$

Where $T_{w}$ is the wall temperature, and $T_{g}$ is the gas inside cylinder temperature and $h$ is convective heat transfer coefficient. The heat transfer rate change with crank angle is given by Hough (1995):

$\frac{\partial Q}{\partial \theta}=-\frac{h A}{\omega}\left(T_{g}-T_{W}\right) \frac{\pi}{180}$

Where $\omega$ is the engine speed.

To calculate convective heat transfer coefficient we use Woschni correlation.

$h=3.26 B^{-0.2} P^{0.8} T^{-0.55} v^{0.8}$

Where, $p$ is instantaneous cylinder pressure, $T$ is instantaneous gas temperature and $v$ is the characteristic velocity of gases.

The characteristic velocity of in cylinder gas for a four stroke engine without swirl is

$v=c_{1} S_{p}+c_{2} \frac{V_{d} T}{P V}\left(p-p_{m}\right)$

The constants in equation (14) are as below:

$$
\begin{array}{llc}
-180 \leq \theta \leq \theta_{0} & c_{1}=2.28 & c_{2}=0 \\
\theta_{0} \leq \theta \leq+180 & c_{1}=2.28 & c_{2}=3.24 \times 10^{-3}
\end{array}
$$

$P_{m}$ is the motoring pressure that can be calculated from equation (15):

$p_{m}=\frac{\left[\left(\frac{C r V_{d}}{C r-1}\right)^{\gamma} p\right]}{V^{\gamma}}$

\section{Mass flow rate equation}

The mass flow rate through a poppet valve is usually described by the equation for the compressible flow through a flow restriction. This equation is derived from one dimensional isentropic flow analysis and the real gas flow effects are included by means of an experimentally determined discharge coefficient, $C_{D}$.

The mass flow rate is related to the upstream stagnation pressure $P_{0}$ and stagnation temperature $T_{0}$, static pressure just downstream the flow restriction $P_{T}$ and a reference area $A_{R}$ characteristic of the valve design and it is represent as below (Navarro et al., 2007):

$$
\dot{m}=\frac{C_{D} A_{R} P_{0}}{\left(R T_{0}\right)^{0.5}}\left(\frac{P_{T}}{P_{0}}\right)^{\frac{1}{\gamma}}\left\{\frac{2 \gamma}{\gamma-1}\left[1-\left(\frac{P_{T}}{P_{0}}\right)^{\frac{\gamma-1}{\gamma}}\right]\right\}^{0.5}
$$

When the flow is choked, $\frac{P_{T}}{P_{0}} \leq\left[\frac{2}{\gamma+1}\right]^{\frac{\gamma}{\gamma-1}}$ the appropriate equation is 


$$
\dot{m}=\frac{C_{D} A_{R} P_{0}}{\left(R T_{0}\right)^{0.5}}(\gamma)^{\frac{1}{2}}\left\{\frac{2}{\gamma+1}\right\}^{\frac{\gamma+1}{2(\gamma-1)}}
$$

For the flow into the cylinder through an intake valve, $P_{0}$ is the intake system pressure and $P_{T}$ is the cylinder pressure. For flow out of the cylinder through an exhaust valve, $P_{0}$ is cylinder pressure and $P_{T}$ is the discharge system pressure. The value of $C_{D}$ for both intake and exhaust valves is taken 0.7 as proposed by Sun and Ren (1995) and the reference area $A_{R}$ is the valve head area.

Piston movement and Volume of the reciprocating compressor equation

Fig. 1 Reciprocating compressor geometric properties

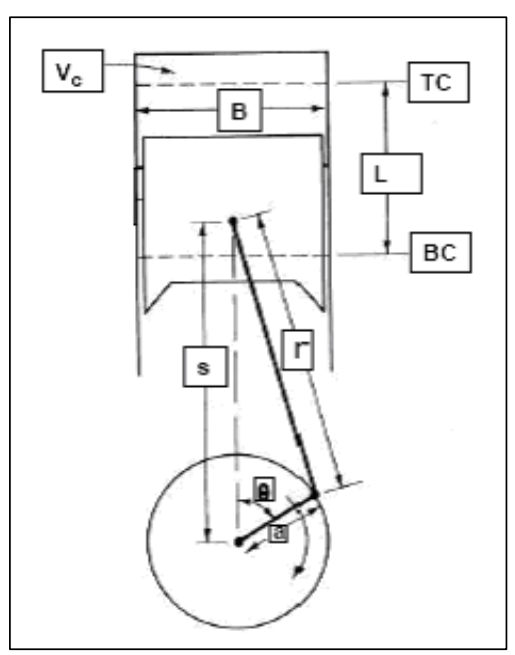

Top dead center being the origin of coordinates of the piston displacement as shown in Fig 1, the instantaneous position of the piston displacement may be given by:

$L(\theta)=a+r-$

$\left[\begin{array}{l}\left(r^{2}-a^{2} \sin ^{2} \theta\right)^{\frac{1}{2}}+ \\ a \cos \theta\end{array}\right]$

Where a is crank radius and $r$ is connection rod length and $L$ is Stroke equal twice crank radius.

The swept volume of reciprocating compressor is given by:

$V_{d}=V_{T C}-V_{B C}$

$V_{d}=\frac{\pi}{4} B^{2} L$

Where $B$ is the piston diameter.

The volume of compressor cylinder and the rate of its change can be expressed respectively, by

$V(\theta)=\frac{\pi}{4} B^{2} L(\theta)$

$\frac{d V}{d \theta}=\frac{1}{2} V_{d} \sin (\theta)\left(1+\cos (\theta)\left(R^{2}-\sin ^{2}(\theta)\right)^{-0.5}\right)$

$R=\frac{r}{a}$

We can see the total volume in cylinder in equation (20)

$V_{t}(\theta)=V(\theta)+V_{c}$

We can conclude the surface area change with crank angle in equation (21).

$$
A=\frac{\pi}{2} B^{2}+\pi B L(\theta)
$$

ISSN: 0974- 6846

\section{Numerical solution}

Among the above equations, the main equations for simulation process are the energy equation, the gas equation of state, and the mass flow continuity equation. The heat exchange equation is absorbed into the energy equation.

The displacement equation of piston is introduced to determine the changing law of medium state parameter in the cylinder whit time.

Differential equations to be solved are constituted from Eqns (5), (10) are usually solved by a numerical method.

In the present work, a standard 4th order Runge-Kutta method was used. Thus, all the above differential equations become 1st order ordinary differential equation. In this work $\Delta t=0.02$ and $\Delta x=0.001$.

\section{Exergy Analysis}

For an open system experiencing mass exchange with the surrounding environment, the following equation holds for the total availability on a time basis.

$$
\frac{d A_{c v}}{d t}=E_{Q}-\left(\dot{W}-P_{0} \frac{d_{c v}}{d t}\right)+\sum_{\text {in }} \dot{m}_{\text {in }} \zeta_{\text {in }}-\sum_{\text {out }} \dot{m}_{\text {out }} \zeta_{\text {out }}-\dot{I}_{c v}
$$

Many of process consider in engineering are steady state ones (such as compressor), which means that the condition in control volume do not change with time, and the eqn (22) can be simplified to

$0=E_{Q}^{\cdot}-\dot{W}+\sum_{\text {in }} \dot{m}_{\text {in }} \zeta_{\text {in }}-\sum_{\text {out }} \dot{m}_{\text {out }} \zeta_{\text {out }}-\dot{I}_{c v}$

In eqn (23), $E=\int\left(1-\frac{T_{0}}{T_{j}}\right) Q_{j}$ is the availability term for heat transfer, with $T_{j}$ the temperature at the boundary of system, and $\dot{Q}_{j}$ represent the time rate of heat transfer at the boundary of the control volume. $\dot{W}$ is the availability term associated with work transfer. $\sum \dot{m} \zeta$ is the availability term associated with inflow and outflow of masses. In particular, the terms $\zeta$ in eqn (23) refer to the flow or stream availability (or exergy) of the incoming and the outgoing cylinder mass flow rates, given by (neglecting kinetic and potential energy contribution):

$\zeta=h-h_{0}-T_{0}\left(s-s_{0}\right)$

$I$ is the rate of irreversibility.

When consider the control volume is the whole compressor we can rewrite the eqn (23) as bellow:

$0=E_{Q}-\dot{W}_{e}+\sum_{\text {in }} \dot{m}_{\text {in }} \zeta_{\text {in }}-\sum_{\text {out }} \dot{m}_{\text {out }} \zeta_{\text {out }}-\dot{I}_{\text {tot }}$ 
Where, $\dot{W}_{e}$ is electrical work, and $\dot{I}_{\text {tot }}$ is total rate of irreversibility, and consider the compressor outer surface is isolated.

If the control volume is the gas inside cylinder, we can rewrite the eqn (23) as bellow:

$0=E_{Q_{j}}-\dot{W}_{c v}+\sum_{\text {in }} \dot{m}_{\text {in }} \zeta_{\text {in }}-\sum_{\text {out }} \dot{m}_{\text {out }} \zeta_{\text {out }}-\dot{I}_{f}$

Where, $\dot{I}_{f}$ is fluid rate of irreversibility.

With combining equation (25) and (26) and arranging it we get

$\dot{W}_{c v}=-E_{Q_{j}}+\dot{W}_{e}-\left(\dot{I}_{t o t}-\dot{I}_{f}\right)$

By using brake work we can calculate brake thermal efficiency, the friction mean effective pressure is calculated using the formula introduced by branes-moss [12] as:

FMEP $($ bar $)=0.97+0.15(\mathrm{rpm} / 1000)+0.05(\mathrm{rpm} / 1000)^{2}(28)$

The mechanical efficiency by exergy analysis is given by:

$\eta_{m}=\left(\frac{W_{\text {cycle }}}{W_{e}}\right)$

Energy balance:

$\frac{d U_{c v}}{d t}=\sum \dot{Q}-\dot{W}+\sum_{\text {in }} \dot{m}_{\text {in }} h_{\text {in }}-\sum_{\text {out }} \dot{m}_{\text {ou }} h_{\text {out }}$

Where $\mathrm{h}$ is flow enthalpy per unit mass at the relevant temperature and pressure.

We can rewrite equation (14-18) and replace $\zeta$ and $E_{Q}$ with $\mathrm{h}$ and $\sum \dot{Q}$ respectively for energy analysis:

The mechanical efficiency by energy analysis is given by:

$\eta_{m}=\left(\frac{W_{\text {cycle }}}{W_{e}}\right)$

Fig. 2. Effect of engine speed on in-cylinder pressure

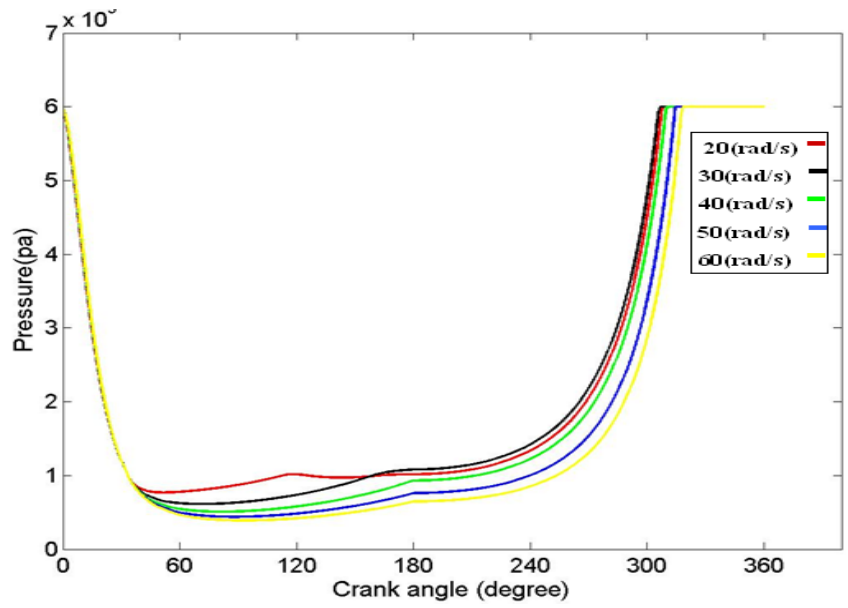

Vol. 5 No. 3 (Mar 2012)

ISSN: 0974- 6846

Fig.3. Effect of engine speed on in-cylinder pressure

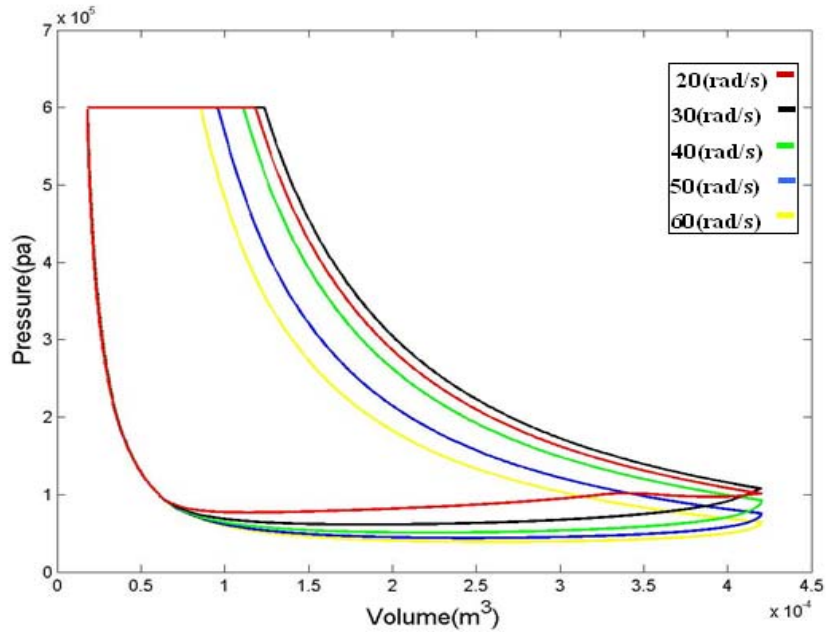

Fig. 4.Effect of engine speed on in-cylinder temperature

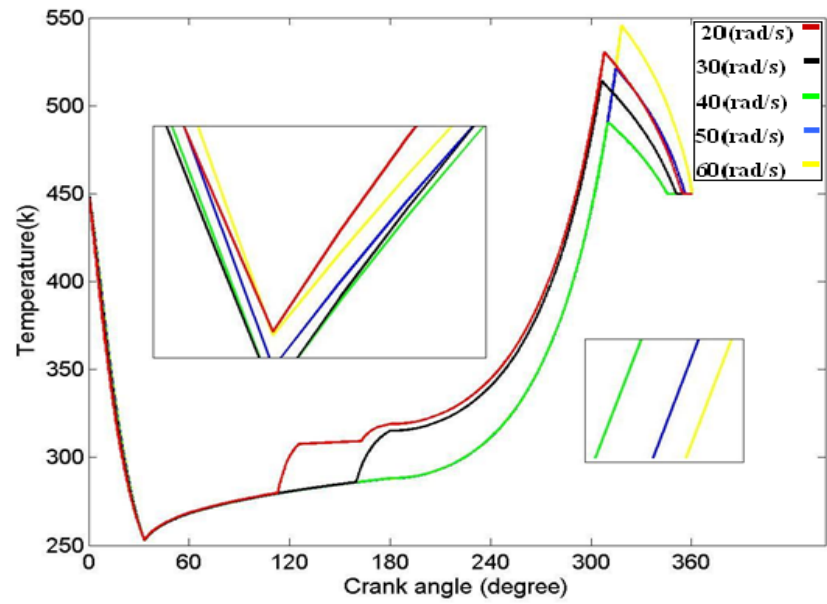

Fig.5. Effect of engine speed on in- cylinder Mass

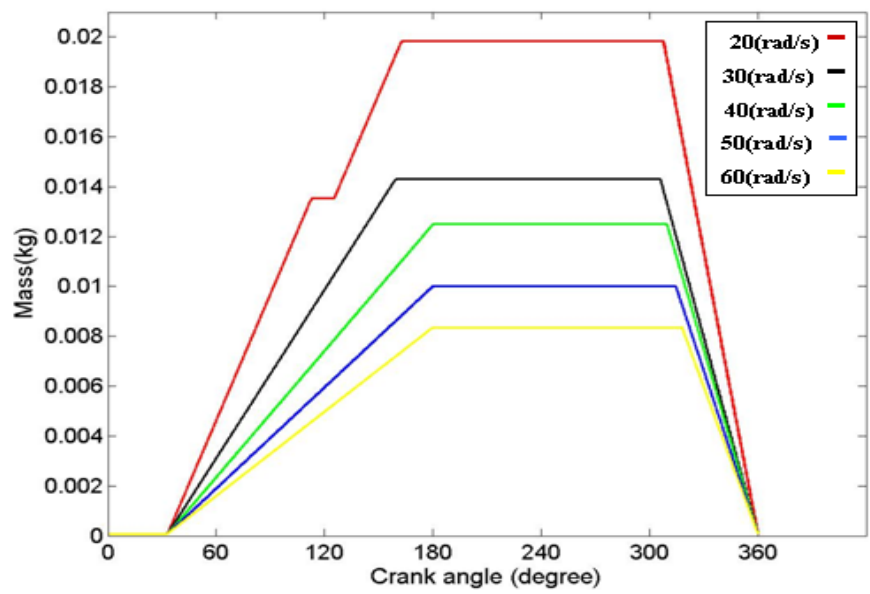


Fig.6. Exergy absorbed by flow with various speed engine

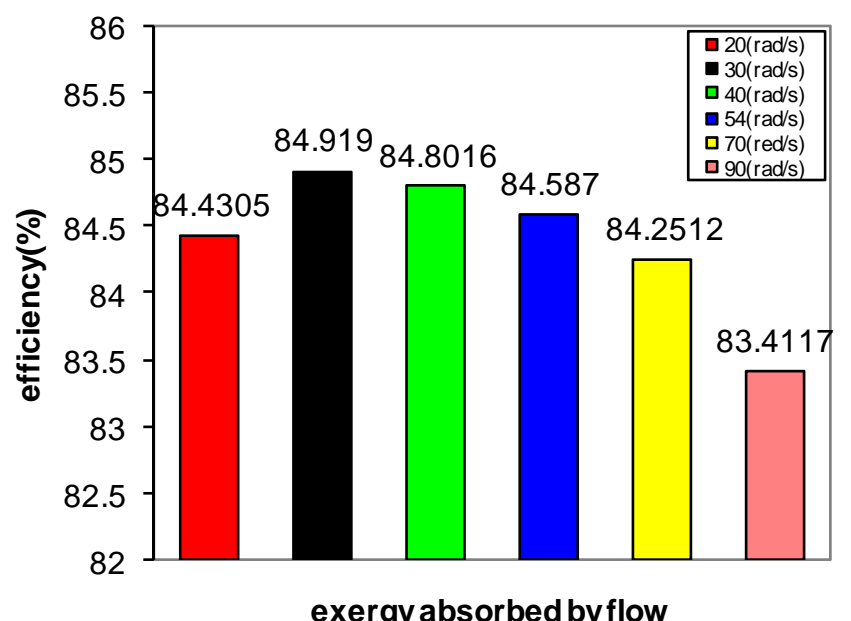

Fig. 7 total exergy destruction with various speed engine

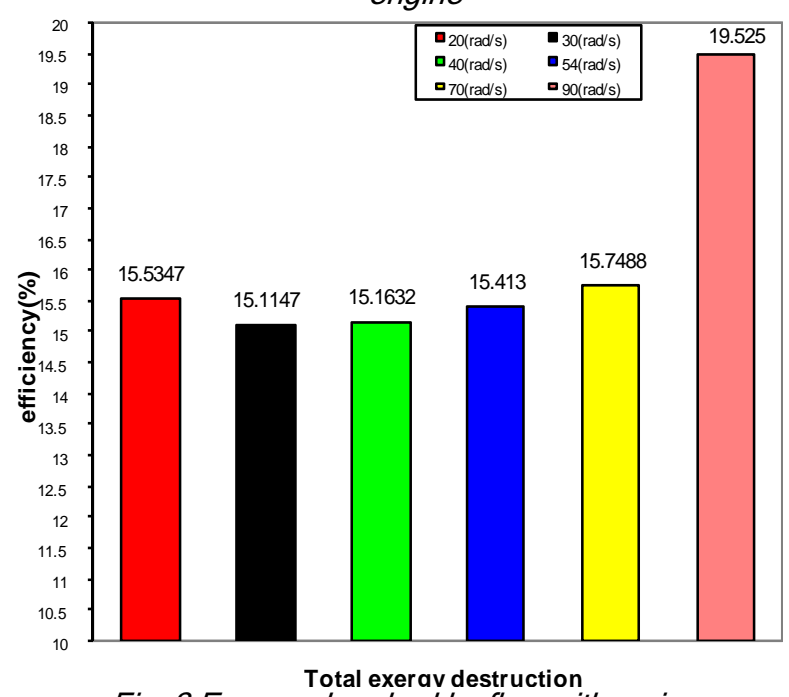

Fig. 8. Energy absorbed by flow with various speed engine

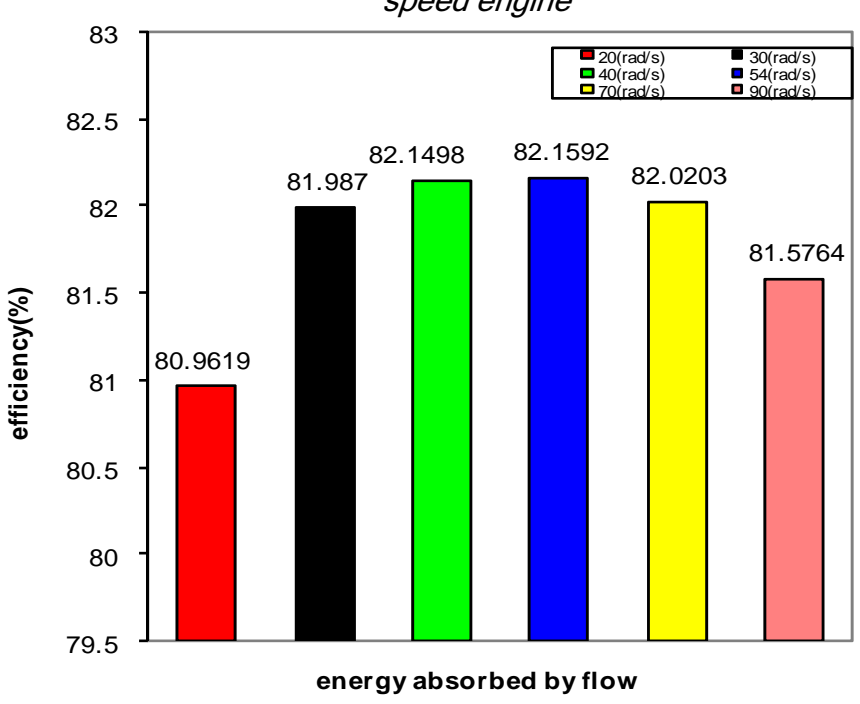

Fig.9. Total energy destruction with various speed engine

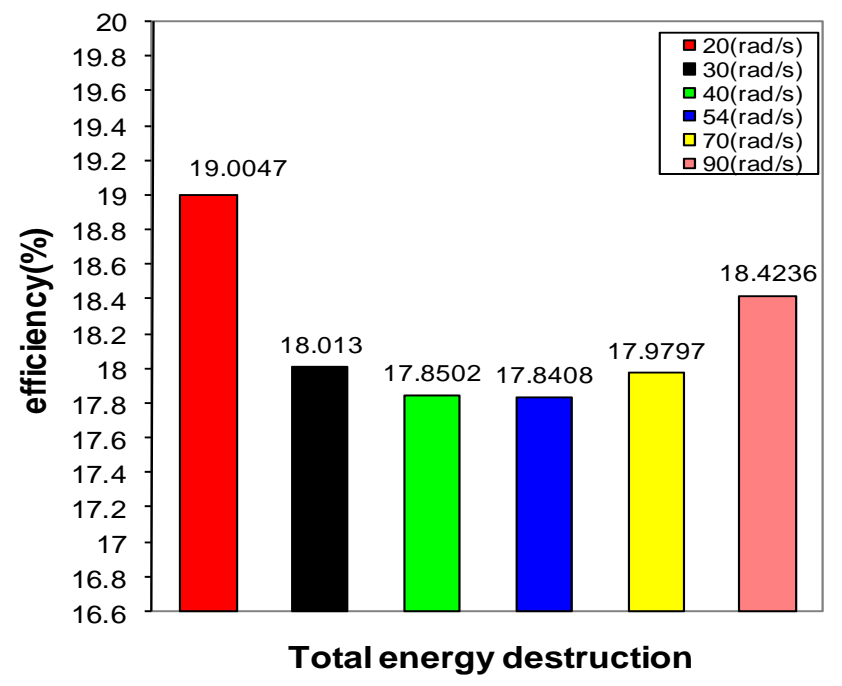

Fig. 10. Efficiency with energy and exergy analysis

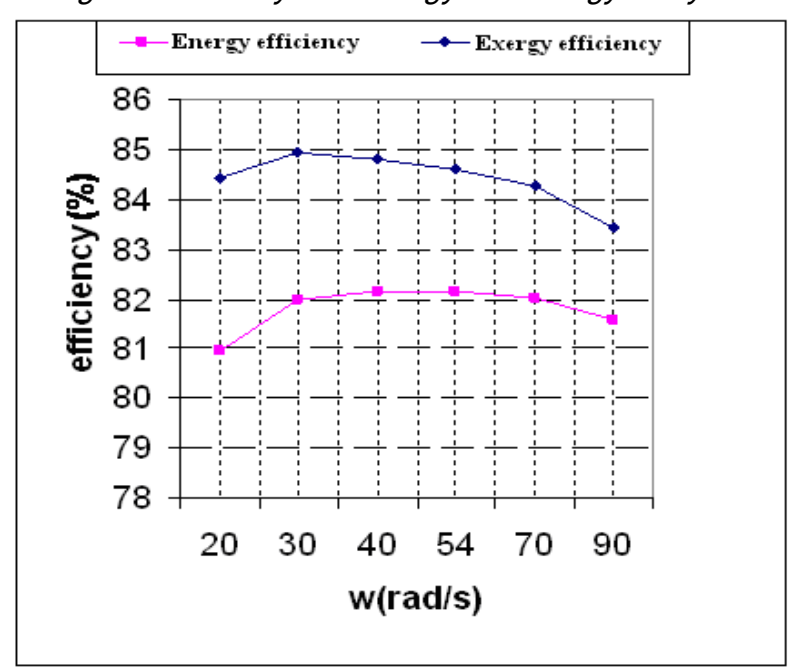

Results and discussion

Fig. 2 and 3 shows effects of engine speed on incylinder pressure. It can be seen that the pressure increases as speed increases. The result has good agreement with the actual value. Fig. 4 shows the effect of engine speed on -cylinder pressure. It can be seen that the temperature decreases as speed increases. This is due to larger heat lost to the cylinder walls as available time for heat transfer increases.

In this work for inlet and outlet flow we have two non return valves. In $\mathrm{w}=20 \mathrm{rad} / \mathrm{s}$ when crank angle more than 100 deg the pressure inside cylinder over than one atmosphere and valve closed and suction complete without mass flow rate then the temperature has extra increase .

The same discus is true for $w=30 \mathrm{rad} / \mathrm{s}$. Fig. 5 show the effect of engine speed on in mass flow rate. It can be seen that the mass decrease as speed increases. Fig. 6 show the exergy absorbed by flow with various engine speed. When engine speed $=30 \mathrm{rad} / \mathrm{s}$ the exergy 
absorbed is maximum. Fig. 7 shows the exergy destruction with various engine speed. When engine speed $=30 \mathrm{rad} / \mathrm{s}$ the exergy destruction is minimum. Fig. 8 show the energy absorbed by flow with various engine speed. When engine speed $=54 \mathrm{rad} / \mathrm{s}$ the energy absorbed is maximum. Fig. 9 shows the energy destruction with various engine speeds. When engine speed $=54 \mathrm{rad} / \mathrm{s}$ the energy destruction is minimum. Fig. 10 shows the efficiency with energy and exergy analysis. Conclusion

In this paper a simple thermodynamics model has been developed to study the effect of engine speed on thermodynamics properties of a typical compressor. The model can predict the volumetric effectiveness, the specific work and the indicated efficiency of the reciprocating compressor for different operating pressure ratios. The model allows physical interpretation. In particular, the influence wall heat transfers is dealt with and the negative impact of the clearance volume is evaluated

\section{References}

1. Cezar OR, Raul H and Diogo EV (2011) A semiempirical model for the unsteady-state simulation of reciprocating compressors for household refrigeration applications. Appl. Thermal Engg. 31(6), 1114-1124.

2. Duprez $M$, Dumont $E$ and Frère $M$ (2007) Modelling of reciprocating and scroll compressors. Int. J. Refrig. 30(5), 873-886.

3. Gorji-Bandpy M and Goodarzian H (2011), Exergoeconomic optimization of gas turbine power plants operating parameters using genetic algorithms: a case study. Thermal Sci. J. 15(1), 4354.

4. Gorji-Bandpy M, Goodarzian H and Biglari M (2010) The Cost-effective analysis of a gas turbine power plant.energy sources, Part B: Economic. Planning \& Policy. 5(4), 348-358.

5. Heywood JB (1988) Internal Combustion Engine Fundamentals. McGraw-Hill, Inc.

6. Hough D (1995) The design, selection, and application of reciprocating compressors for fuel gas service. J. Engg. Gas Turbines Power. 117, 88-93.

7. Navarro EE, Granryd E, Urchueguía JF and Corberán JM (2007) A phenomenological model for analyzing reciprocating compressors. Int. J. Refrig. 30(7), 12541265.

8. Ndiaye D and Bernier M (2010) Dynamic model of a hermetic reciprocating compressor in on-off cycling operation (Abbreviation: Compressor dynamic model). Appl. Thermal Engg. 30(9), 792-799.

9. Seyyedi SM, Ajam A and Farahat SA (2010) New Approach for optimization of thermal power plant based on the exergoeconomic analysis and structural Optimization method: Application to the CGAM Problem. Energy Conversion \& Manage. 51(11), 2202-2211.
10. Stouffs P, Tazerout $M$ and Wauters P (2001) Thermodynamic analysis of reciprocating compressors. Int. J. Therm.Sci. 40(2), 52-66.

11. Sun SY and Ren TR (1995) New method of thermodynamic computation for a reciprocating compressor: computer simulation of working process. Int. J. Mech. Sci. 37, 343-353.

12. Tsatsaronis $G$ (2007) Definition and nomenclature in exergy analysis and exergoeconomics. Energy. 32(4), 249-253.

13. Weclas M, Melling A and Durst F (1998) Flow separation in the inlet valve gap of piston engines. Prog. Energy Combustion. Sci. 24, 165-195. 